\title{
A shopping trolley seismograph
}

SIR - On 17 January 1995, a devastating earthquake struck the port city of Kobe in western Japan, leaving 5,502 people dead and 2 missing. The catastrophic scenes were recorded at many 'convenience' stores by closed-circuit video cameras. These video records (kindly provided by $\mathrm{K}$. Nishimura of the Japanese Broadcasting Corporation (NHK) Kobe office) enable us to estimate ground motion and thereby to infer the nature of the buried fault beneath Kobe. Its precise location is still vague, although the southwestern part of the fault was traced along a pre-existing fault on Awaji Island (Nakata, T. et al. J. Geogr. 104, 127-142; 1995). The convenience stores usually use basket-stands with castors which can move
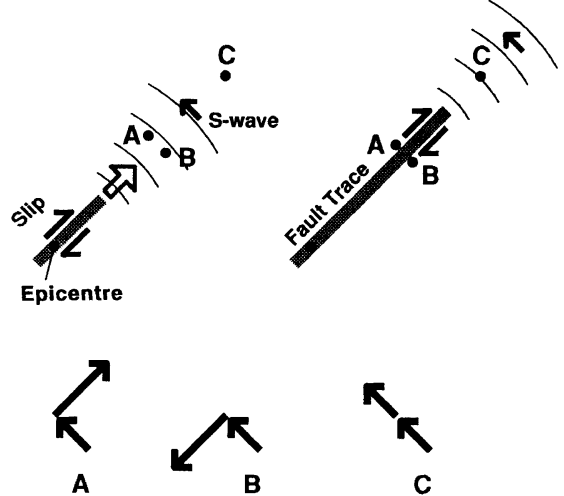

FIG. 1 Idealized ground motion associated with right-lateral fault propagation. Points $A$ and $B$ are located on opposite sides of an extending fault trace, while $C$ is beyond the fault tip. These points were all shaken to the northwest by $S$ waves generated from the epicentre, and then subjected to different motion depending on their location relative to the fault trace. The combination of $S$ wave and the subsequent motion gives three different patterns of ground motions as shown at the bottom in any direction on the floor. According to the inertia law, the basket-stands must move in the opposite direction to the motion of the floor, allowing their use as a form of seismograph.

A typical scene appearing on the video display is as follows. People first noticed the P-wave arrival through the vibration of the floor and other objects. A few seconds later, a large horizontal motion occurred. This later motion is interpreted as the arrival of the $\mathrm{S}$ wave. Soon after this, a larger shock hit stores. This larger shock can be interpreted as the direct effect of the fault slip beneath the stores.

The basic idea for inferring the surface trace of the buried fault is illustrated in Fig. 1. There are three theoretical patterns of ground motion depending on the location of the stores relative to a propagating, right-lateral fault: (1) on the north side of the fault the ground moves towards the northwest and then northeast, effectively moving clockwise; (2) on the south side of the fault the ground moves towards the northwest and then southwest, effectively moving anticlockwise; (3) at the extension from a fault tip the ground moves toward the northwest only.

The actual ground motions were more complex than these simple patterns, probably due to heterogeneous slip on several fault segments. Moreover, video records were often stopped immediately after the $S$ wave's arrival because of the interruption of the electricity supply. In spite of these difficulties, these basic patterns were identified for nearly half of the video records obtained. In addition to these data, two more sets of data are available. One is the video record at the Kobe office of NHK. The other is GPS

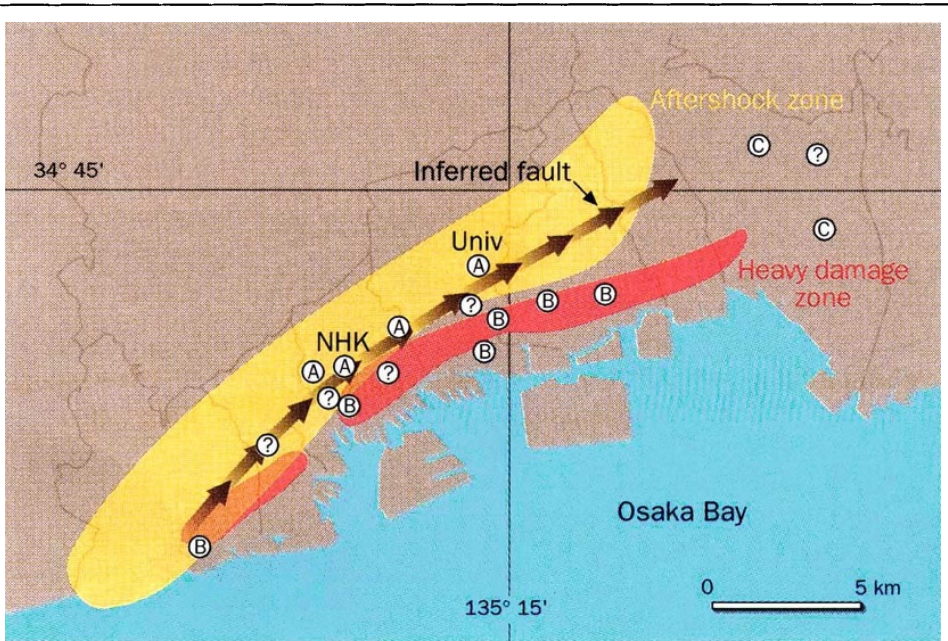

FIG. 2 Map of the Kobe area. Ground motion patterns (A, B and C) identified by the video data are plotted at the sites of the convenience stores. 'Univ' denotes Kobe University; '?' indicates that the pattern cannot be identified because the video records were stopped immediately after S-wave arrival. The solid line with arrows indicates a fault trace inferred from these data as well as the teleseismic waveform analysis. data at Kobe University. Both locations are inferred to be on the north side of a fault trace. The strong-motion instrument at Kobe JMA (Japan Meteorological Agency), which is a little west of NHK, also recorded the ground displacement. It roughly indicates a clockwise motion following the arrival of the lateral $\mathrm{S}$ wave.

The ground motion patterns are summarized in Fig. 2. The video data alone cannot well constrain the northeastern part of the fault trace. For this part the information obtained from teleseismic body-wave analysis was used, where three sub-events with different fault orientations were derived (Kikuchi and Kanamori, unpublished data). The fault strike of the third sub-event, which is located in the Kobe area, is $\mathrm{N} 60^{\circ} \mathrm{E}$.

This study shows that the earthquake fault co-seismically reached the area beneath Kobe and that large opposite ground motions occurred at two adjacent points, only $1 \mathrm{~km}$ apart (NHK and the southern point). This enables us to pinpoint the earthquake fault beneath Kobe (Fig. 2). The fault trace (that is, the intersection of the fault plane with the surface) runs between the heavily damaged zone and the aftershock zone.

Thus, video records at convenience stores can be very usefully employed as a form of seismogram. They also contain useful information about human behaviour, providing us with lessons on how to protect ourselves during sudden, strong earthquake shocks.

Masayuki Kikuchi

Physics Department,

Yokohama City University, Seto,

Kanazawa-ku, Yokohama 236, Japan

\section{Olfactory receptors guide axons}

SIR-Subsets of olfactory receptor neurons (ORNs), each expressing a particular type of receptor protein $^{1,2}$, are broadly distributed in the sensory epithelium ${ }^{3,4}$; yet members of each subset send their axons to only one or a few of some 2,000 modules, called glomeruli, in the olfactory bulb $^{5,6}$. The basis for this specific axon targeting is unknown.

A clue has come from correlated mutation analysis, which has identified residues in the receptors that may be critical for binding odour molecules ${ }^{7}$. This has supported previous evidence that odour molecule determinants interact differentially with residues within a transmembrane binding pocket in the receptors ${ }^{8}$. In addition, we have found that specific residues in the second extracellular loop (E2) show correlations with critical residues in the binding pocket (see figure), implying a functional relation between these residues in the loop and the binding pocket ${ }^{7,9}$. 